\title{
Ethnic effects when facing children: An ERP study
}

\author{
Hannah Spencer ${ }^{\mathrm{a}}$, Ivo Heitland ${ }^{\mathrm{b}}$, Estrella R. Montoya ${ }^{\mathrm{a}}$, Susan J.T. Branje ${ }^{\mathrm{c}}$, Peter A. Bos ${ }^{\mathrm{a}, *}$ \\ a Utrecht University, Department of Experimental Psychology, Heidelberglaan 1, 3584CS, Utrecht, The Netherlands \\ b Hannover Medical School, Department of Psychiatry, Social Psychiatry and Psychotherapy, Carl-Neuberg-Straße 1, D-30625, Hannover, Germany \\ ${ }^{\mathrm{c}}$ Utrecht University, Research Centre Adolescent Development, Department Youth \& Family, Heidelberglaan 1, 3584 CS, Utrecht, The Netherlands
}

\section{A R T I C L E I N F O}

\section{Keywords:}

Intergroup effects

Facial processing

Child faces

EEG

N170

N200

\begin{abstract}
A B S T R A C T
Abundant research has highlighted a disadvantage experienced by children of ethnic minority groups in, for example, educational and health care settings. In order to understand implicit attitudes that contribute to ethnic disparities, underlying neural correlates have been widely studied. However, this has been limited to the context of adults. Using a sample of nulliparous Caucasian females $(N=46)$, the current study is the first to examine how early attentional and facial perceptual processing stages, assessed with event-related brain potentials (ERPs), differentiate for stimuli of young ingroup (of the same ethnicity) or outgroup (of a different ethnicity) children. Additionally, we assessed how a differentiation in ERPs may relate to subsequent adult responsiveness to children by measuring both cuteness ratings and motivation to view child faces. Similar to previous findings for adult facial stimuli, we found significant differences in attentional (N200) and facial perceptual (N170) processing when adults were faced with children of different ethnicities. Furthermore, increased differentiation in attentional processing (N200) for ingroup and outgroup children was associated with reduced cuteness ratings of outgroup children. Importantly however, participants showed no overall preference for ingroup child faces, as motivation to view child faces was even greater towards outgroup child faces. In addition, increased self-reported motivation for parental care was related to enhanced cuteness appraisals of outgroup child faces. Taken together, these findings reveal how early social categorization processes may lead to biased behavior when interacting with children of ethnic minorities.
\end{abstract}

\section{Introduction}

Human children are dependent on care by adults for an extensive period of time; not only for basic feeding and protection, but also for guidance in the development of cognitive, emotional, and social skills (Bornstein, 2015). With the growing diversity of populations worldwide due to globalization, children are increasingly dependent on multiethnic interactions with adults in, for example, educational and health care settings. Compelling evidence, however, demonstrates ethnic disparities affecting children in these environments, with children of ethnic minority groups experiencing a disadvantage both in schools, reflected in teachers' expectations and judgments (Bates \& Glick, 2013; Glock, Krolak-Schwerdt, Klapproth, \& Böhmer, 2013; Rubie-Davies, Hattie, \& Hamilton, 2006; Tenenbaum \& Ruck, 2007) and quality of health care (Flores, 2010). It is of great importance to understand implicit attitudes that may contribute to ethnic disparities when adults face children of different ethnicities. In the current study we have therefore assessed how ethnic facial cues may affect adult responses towards children and for the first time examined the neural responses that may underlie this effect.

In order to understand implicit attitudes that contribute to ethnic disparities, neural correlates underlying social categorization, stereotyping, and prejudice have been widely studied (for a review see Ito \& Bartholow, 2009). The focus of these studies, however, has been on social group effects arising in the context of adults; comparable research focusing on adults interacting with children has so far received no attention. Therefore, the current study explored ethnic effects in electrophysiological responses to young child faces. Differences in event-related potential (ERP) patterns measured with electroencephalography (EEG) have repeatedly been revealed in response to adult stimuli of different social groups and are suggested to reflect differences in rapid categorization processes (Ito \& Bartholow, 2009). Facial cues reflective of different ethnic groups appear to affect both early attentional processing stages and subsequent facial perceptual processing stages (Ito \& Bartholow, 2009). Very early attentional ERP components, measured with $\mathrm{N} 100$ and P200 amplitudes, are generally more enhanced in response to outgroup facial stimuli and are suggested to reflect early orientation processes to more novel targets (Correll,

\footnotetext{
* Corresponding author.

E-mail address: p.a.bos@uu.nl (P.A. Bos).
} 
Urland, \& Ito, 2006; Ito \& Bartholow, 2009; Lipp, Mallan, Martin, Terry, \& Smith, 2011). The subsequent N200 component on the other hand is shown to be greater in response to ingroup facial stimuli and is interpreted to reflect a spontaneous greater attention allocation towards more familiar individuals (Balas \& Nelson, 2010; Correll et al., 2006; Derks, Stedehouder, \& Ito, 2015; Ito \& Bartholow, 2009; Lipp et al., 2011; Willadsen-Jensen \& Ito, 2015). Interestingly, studies on early facial processing measured with the N170 component show mixed results, which may be due to differences in experimental set-ups (Ofan, Rubin, \& Amodio, 2011). Some studies reported that outgroup faces elicited greater N170 amplitudes compared to ingroup faces (Balas \& Nelson, 2010; Caharel et al., 2011; Herrmann et al., 2007; Ofan et al., 2011; Stahl, Wiese, \& Schweinberger, 2008; Walker, Silvert, Hewstone, \& Nobre, 2007). Other studies reported that the N170 was not sensitive to the ethnicity of faces (Caldara et al., 2003; Caldara, Rossion, Bovet, \& Hauert, 2004; Vizioli, Foreman, Rousselet, \& Caldara, 2011; Wiese, Stahl, \& Schweinberger, 2009), or even stronger towards ingroup compared to outgroup faces (Ito \& Urland, 2005; Ratner \& Amodio, 2013).

Early categorization of one's own social group enables the rapid activation of evaluations and cognitions associated with this group (Ito \& Bartholow, 2009; Ito, Thompson, \& Cacioppo, 2004). This process not only leads to efficient responses to the needs of members of the same social group, or the ingroup, but also underlies the intergroup bias in which own group members are evaluated more favorably than people belonging to a different social group, or outgroup members (Hewstone, Rubin, \& Willis, 2002). Importantly, individual differences in ERP responses have been related to subsequent biased behavior. For example, larger N170 amplitudes towards outgroup targets compared to ingroup targets were associated with a stronger behavioral ingroup bias (Ofan et al., 2011), and the degree of ethnic differentiation in the P200 and N200 components predicted a behavioral bias in which outgroup targets were perceived as more threatening (Correll et al., 2006). Furthermore, intergroup effects in the N200 component have been related to strong identification with an ethnic group (Derks et al., 2015) and cultural stereotypes (Correll et al., 2006). It is therefore suggested that intergroup disparities in ERP responses underlie the application of stereotyping and prejudice (Ito \& Bartholow, 2009).

While the hypothesis that neural responses underlie implicit attitudes towards children of different ethnicities has not yet been explored, behavioral studies have indicated that responses to infant facial cues may be constrained by the ethnicity of the infant. A study by Hodsoll and colleagues (2010) demonstrated that the attentional prioritization of infant faces was limited to ingroup infants. Furthermore, Senese and colleagues (2017) reported that ingroup infants faces were evaluated as more positive than outgroup infants and demonstrated a connection with genetic polymorphisms and early care experiences. However, these findings are contested by other studies reporting no effects of ethnicity on adult responses towards infant faces. For example, a study by Esposito and colleagues (2014) reported no differences in willingness to interact with infants of the same or a different ethnicity. Additionally they used facial skin temperature as a measure of physiological arousal and found increases in arousal towards all infant faces, with no difference between infants of different ethnicities (Esposito et al., 2014). Furthermore, Proverbio and colleagues (2011) reported that infant ethnicity did not affect attentional attraction and Golle and colleagues (2015) showed that preference for infants did not depend on ethnicity. Instead, these studies propose that responses of adults are primarily affected by perceived cuteness of the infant, independent of ethnicity.

Perceptions of cuteness enhance parental responsiveness (Hahn \& Perrett, 2014; Luo et al., 2015), and are activated by a set of certain facial features, such as large eyes, a small nose, and chubby cheeks, a configurations that is known as a baby schema (or 'Kindschenschema'; Lorenz, 2010). Baby schema enhances activation of the mesocorticolimbic system which mediates reward processing, resulting in increased perception of cuteness (Glocker et al., 2009). In general, attractive or cuter infants enhance motivational processing (Parsons, Young, Kumari, Stein, \& Kringelbach, 2011; Yamamoto, Ariely, Chi, Langleben, \& Elman, 2009) and tend to receive preferential treatment or evaluation (Hildebrandt \& Fitzgerald, 1983; Langlois, Ritter, Casey, \& Sawin, 1995). The effect of baby schema furthermore appears to evoke a very early specific neural response reflecting enhanced attentional processing of infant faces compared to adult faces (Hahn et al., 2016; Jantzen et al., 2017; Kringelbach et al., 2008). Effects of baby schema extend beyond infancy to young children (Luo, Li, \& Lee, 2011) and promotes human caregiving regardless of kinship (Glocker et al., 2009). In the current study we used a validated paradigm to assess the effects of ethnicity on adult behavioral responses towards children by measuring both subjective appraisal of cuteness and behavioral motivation to view children (Hahn, Xiao, Sprengelmeyer, \& Perrett, 2013; Parsons et al., 2011; Yamamoto et al., 2009). This enabled us to assess whether the effect of cuteness on adult responses, as demonstrated with infants, may be constrained by ethnicity.

To summarize, with the current study we attempted to extend the current literature on the neural processes underlying ethnic stereotyping and prejudice by assessing how ethnicity can affect physiological and motivational responses when adults are faced with young children. We have examined if ethnicity influences adult responses towards children by measuring cuteness ratings and motivation to view child faces. Concurrently, we assessed if ERP components related to early attentional processing stages (specifically the N100, P200, and N200 components) and facial perceptual processing stages (specifically the N170 component) differed in response to ingroup or outgroup children, similarly as has previously been established in studies using adult faces of different ethnicities. Finally, we determined if a differentiation in early attentional and facial perceptual ERP amplitudes was related to subsequent adult responses to ingroup and outgroup children.

\section{Methods}

\subsection{Participants}

Forty-six nulliparous Caucasian females participated in the study for partial course credit or financial compensation. Only females were included since the current study was part of a series of studies on caregiving behavior prior to motherhood. Participants were recruited using flyers and posters around Utrecht Science Park. The study was conducted in accordance with the latest version of the Declaration of Helsinki and was approved by the local ethical committee. All participants gave written informed consent prior to study participation. Data sets of eight participants were not included in the final analyses due to excessive artifacts in the EEG signal ( $\geq 25 \%$ of trials), yielding a final sample of thirty-eight subjects (mean age $=22.47, S D=3.05$ ). In order to test whether individual differences in activation of the parental care motivational system would relate to our neural and behavioral measures, all participants completed the Parental Care and Tenderness (PCAT) questionnaire (Buckels et al., 2015). The questionnaire was translated into Dutch by author HS and one independent bilingual Dutch and English speaker using forward- and back-translation. Discrepancies were resolved after discussion with author PB.

\subsection{Stimuli and experimental task}

Eighty color photos of child faces selected from the Child Affective Facial Expression set (CAFE; LoBue \& Thrasher, 2015) were used as stimuli. A set of ingroup and outgroup child facial stimuli was composed with the ingroup set containing forty faces of children who were identified as European American and the outgroup set containing forty faces of children who were identified as African American, Latino or Asian. Gender was equally distributed among the two sets. Facial stimuli were selected that were most consistently rated as depicting a 
neutral facial expression (LoBue \& Thrasher, 2015). The age range of depicted children was between three and seven years old, and the average age did not significantly differ between the ingroup $(M=5.35$ years, $S D=0.79)$ and outgroup $(M=5.39, S D=0.76)$ set, $t$ $(78)=.38, p=.71$.

The experimental task consisted of a keypress and a cuteness rating phase modelled after previous studies (Aharon et al., 2001; Parsons et al., 2011; Yamamoto et al., 2009). During the keypress phase participants were told that they would be presented with a number of photos for five seconds each, but that the presentation time of each photo could be manipulated with keypresses. Participants were told that to make presentations last longer or shorter they could press the up or down arrow key of the keyboard as many times as desired. Total presentation time was increased or decreased with each key press according to a previously established formula (Aharon et al., 2001; Yamamoto et al., 2009):

NewTotalTime $=$ OldTotalTime $+\frac{\text { ExtremeTime }- \text { OldTotalTime }}{K}$.

ExtremeTime was $0 \mathrm{~s}$ for keypresses decreasing the presentation time, ExtremeTime was $10 \mathrm{~s}$ for keypresses increasing the presentation time and $\mathrm{K}$ was the scaling constant and set to 40 . This formula leads to a reduced efficacy of presentation time manipulation with each additional key press (Aharon et al., 2001; Yamamoto et al., 2009). A bar was presented to the right of each photo indicating the remaining presentation time and the speed at which this bar decreased in length changed with every keypress (see Fig. 1). Participants were told that the keypress phase was fixed at five minutes and that this duration was independent of their actions, while in reality the total duration time was manipulated by the number of keypresses. This task phase aimed to assess the incentive salience or motivation to view the child faces by measuring the amount of work participants were willing to do in response to each stimulus (Parsons et al., 2011). The dependent variable obtained from the keypress phase was the total viewing time of each stimulus, directly resulting from the number of keypresses.

During the cuteness rating phase participants rated the cuteness of the child faces using a visual analogue scale. Ratings were given on a scale from -4 'Not cute' to +4 'Very cute'. Similar to the keypress phase,

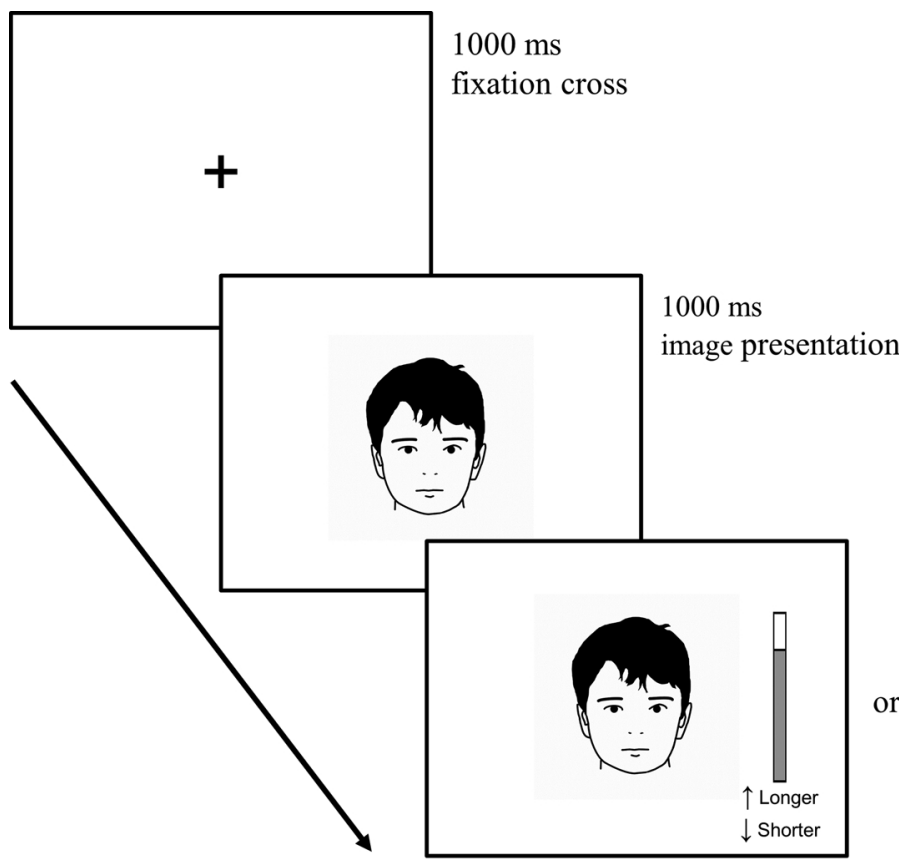

Duration wanting phase trail dependant on keypresses a bar was presented to the right of the stimulus which now represented a visual analogue scale that participants could manipulate in length by pressing the up and down arrow keys on the keyboard. Each child face was presented for a set duration of $5 \mathrm{~s}$ (see Fig. 1).

Participants first completed the keypress phase followed by the cuteness rating phase. Two stimuli subsets of forty child faces were composed with equal distributions of gender and ethnic group. In order to avoid differential subset effects, these two subsets were counterbalanced over both phases across participants. EEG data was recorded continuously during task execution. Each trial of both task phases started with a fixation cross $(1000 \mathrm{~ms})$ after which the stimuli independently were presented on screen for one second prior to bar presentation. Participants were explicitly instructed to only respond once the bar appeared. This enabled ERP data collection unconfounded by motor responses.

\subsection{Electroencephalography (EEG) data collection and reduction}

EEG data recordings were made using the Biosemi ActiveTwo system (Biosemi, Amsterdam, The Netherlands) at $2048 \mathrm{~Hz}$ sampling rate from $5 \mathrm{~A} \mathrm{G} / \mathrm{AgCl}$ pin electrodes placed over three midline $(\mathrm{Fz}, \mathrm{Cz}$, and $\mathrm{Pz}$ ) and two lateral posterior (P7 and P8) scalp locations according to the International 10/20 EEG system. Scalp locations were selected based on previous research indicating that social group effects in N100, P200, and N200 components occur maximally over midline scalp locations (e.g. Derks et al., 2015; Ito et al., 2004; Kubota \& Ito, 2007), while the face-sensitive N170 component occurs maximally over lateral posterior locations (Eimer, 2011). The ground reference point consisted of the active common mode sense (CMS) and passive driven right leg (DRL) electrode. Reference electrodes were placed over the left and right mastoid. Vertical eye movements were measured with electrodes placed above and below the left eye.

Raw EEG traces were re-referenced to the average of the left and right mastoids and down-sampled to $256 \mathrm{~Hz}$. Data were $0.1-30 \mathrm{~Hz}$ band pass filtered, with a $24 \mathrm{~dB}$ roll-off per octave and a notch filter of $50 \mathrm{~Hz}$. Data were segmented ( $100 \mathrm{~ms}$ pre-stimulus $-800 \mathrm{~ms}$ post-stimulus) and baseline corrected relative to a $100 \mathrm{~ms}$ pre-stimulus interval. Segments containing eye blinks were rejected through semi-automatic inspection

Fig. 1. Task design of the keypress and cuteness rating phase. A $1000 \mathrm{~ms}$ fixation cross was followed by a $1000 \mathrm{~ms}$ presentation of a child face after which a bar would be presented to the right of the stimulus. During the keypress phase the bar represented the remaining presentation time of the stimulus which could be manipulated by keypresses. During the cuteness rating phase the bar represented a visual analogue scale of the rating of the stimulus to the right of the stimulus. Participants could manipulate the length of the bar by pressing the up or down arrow keys reflecting a higher or lower rating. Above image is used as illustration, during task execution child face stimuli consisted of original images from the CAFE set (LoBue \& Thrasher, 2015). 


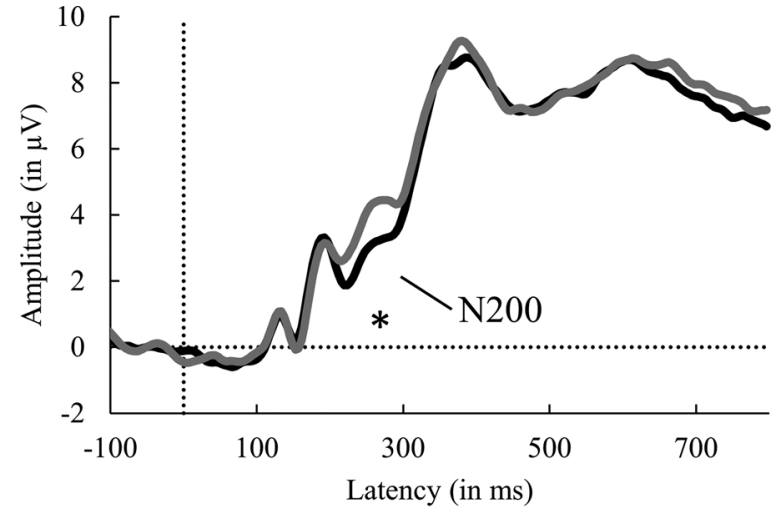

Ingroup $\longrightarrow$ Outgroup

Fig. 2. Grand averaged ERP-waveforms at the midline parietal electrode for ingroup and outgroup child faces collapsed over both task phases. *significant difference in N200 amplitude at $\mathrm{p}<.05$.

of trials with maximal allowed amplitudes of $\pm 150 \mu \mathrm{V}$. Additional artifacts were rejected through semi-automatic inspection of EEG channels, with maximal allowed amplitude differences of $100 \mu \mathrm{V}$ within a segment and lowest allowed activity of $0.5 \mu \mathrm{V}$ in $100 \mathrm{~ms}$ intervals. On average, artifacts were detected in $9.93 \%$ of all trials per participant. Segments were averaged into the ingroup and outgroup conditions separately for the keypress and cuteness rating phase, resulting in a total of four conditions per participant.

Statistical analyses were performed for three ERP components measured over midline electrodes: the N100, P200, and N200. The N170 component was measured over the lateral parietal electrodes. All components were quantified by computing the average voltage over a 50-ms time window centered on the peak latencies of the respective ERP components. Peak latencies of the ERP components were detected in the grand averaged waveforms of all trials combined (Luck, 2014). This resulted in the following time windows: $127-177 \mathrm{~ms}$ for the N100; $169-219$ ms for the P200; and 170-220 ms for the N170. Through visual inspection it was determined that a time window of $50 \mathrm{~ms}$ would not be sufficient to cover the N200 component, since the N200 had a wider configuration compared to the earlier N200, P200, and N170 components. Therefore, the N200 was determined by computing the average voltage over a broader time window of $100 \mathrm{~ms}$ (221-321 ms).

\subsection{Data analysis}

\subsubsection{Behavioral data analyses}

Differences in viewing times and cuteness ratings for ingroup and outgroup child faces were examined with two separate paired t-tests.

\subsubsection{ERP data analyses}

Full-factorial $(3 \times 2 \times 2)$ repeated measures ANOVAs were conducted with within-subject factors of Scalp location (Fz, Cz, and $\mathrm{Pz}$ ), Task phase (Key pressing and cuteness rating phase), and Ethnic group (Ingroup and Outgroup) for the N100, P200, and N200 components. For the N170 component a full-factorial $(2 \times 2 \times 2)$ repeated measures ANOVA was conducted with within-subjects factors of Scalp location (P7 and P8), Task phase (Key pressing and cuteness rating phase), and Ethnic group (Ingroup and Outgroup). $F$ values for ANOVAs of mean amplitudes are reported with uncorrected degrees of freedom and Greenhouse-Geiser corrected $p$ values.

\subsubsection{Relation between ERPs and behavior}

In order to evaluate intergroup effects measured in ERPs on behavior, we calculated difference scores between amplitudes to ingroup and outgroup child faces when ERP data analyses revealed significant effects of ethnic group on specific ERP components. Correlations between ERP difference scores and behavioral measures were examined using two-tailed Pearson's correlation analyses. To further test if our behavioral and ERP findings would relate to individual differences in motivation for parental care, we correlated total scores on the PCAT questionnaire with both the ERP difference scores for ERP components with a significant effect of ethnic group, and with the viewing times and cuteness ratings using two-tailed Pearson's correlation analyses.

\section{Results}

\subsection{Behavioral results}

During the keypress phase, the viewing time in seconds of outgroup child faces was significantly longer $(M=5.07, S D=0.42)$ compared to ingroup child faces $(M=4.96, S D=0.36), t(37)=2.54, p=.02, \eta^{2}$ $=0.15$. This indicated that incentive salience, or amount of work participants were willing to do to view child faces, was greater for outgroup compared to ingroup child faces.

There was no significant difference in cuteness ratings between ingroup and outgroup child faces, $t(37)=1.68 p=.10$, indicating no intergroup bias in cuteness appraisal of children of different ethnicities.

\subsection{ERP results}

\subsection{1. $N 100 \& P 200$}

For both the N100 and P200, analyses revealed no significant effects of ethnic group on amplitudes. There was a significant main effect of electrode for N100 components, $F(2,74)=85.14, p<.001, \eta_{p}{ }^{2}=.70$, and for P200 components, $F(2,74)=60.70, p<.001, \eta_{p}{ }^{2}=.62$. N100 components were largest measured over frontal regions and P200 components were largest measured over parietal regions.

\subsubsection{N200}

Analyses revealed a significant interaction between ethnic group and electrode, $F(2,74)=3.75, p=.04, \eta_{p}{ }^{2}=.09$. Separate analyses per electrode revealed significant effects for $\mathrm{Pz}$, but not for $\mathrm{Fz}$ and $\mathrm{Cz}$. For Pz, there was a significant effect of ethnic group on the N200, F (1, $37)=8.41, p=.006, \eta_{p}{ }^{2}=.19$; N200 amplitudes were significantly greater (more negative) towards ingroup child faces $(M=3.46 \mu \mathrm{V}$, $S D=4.25)$ compared to outgroup child faces $(M=4.35 \mu \mathrm{V}$, $S D=4.05$ ), see Fig. 2. Additionally, there was a significant effect of task phase on the N200 with stronger components during the keypress phase compared to the cuteness rating phase, $F(1,37)=14.95, p<$ $.001, \eta_{p}{ }^{2}=.29$. However, this effect did not qualify the effects of ethnic group since interaction effects were non-significant, $p s>.12$. Finally, there was a significant main effect of electrode for N200 components, $F$ $(2,74)=136.86, p<.001, \eta_{p}{ }^{2}=.79$. N200 components were largest measured over central regions.

\subsection{3. $N 170$}

Analyses showed a significant three-way interaction between ethnic group, electrode and task phase, $F(1,37)=8.26, p=.007, \eta_{p}{ }^{2}=.18$. Separate analyses per electrode revealed a significant effect of ethnic group on N170 amplitudes measured over P7, $F(1,37)=8.29, p=$ $.007, \eta_{p}{ }^{2}=.18$. N170 amplitudes were significantly stronger (more negative) in response to outgroup child faces $(M=2.97, S D=3.57)$ compared to ingroup child faces $(M=4.06, S D=3.72)$, see Fig. 3 . For P7, there were no effect of task phase, nor was the effect of ethnic group qualified by an interaction with task phase, both $p s>.48$. Measured over P8, there was a significant interaction effect between ethnic group and task phase, $F(1,37)=5.02, p=.03, \eta_{p}{ }^{2}=.12$. Post-hoc pairwise comparisons, however, revealed no significant differences between social groups during both the keypress and cuteness rating phases as measured over P8, $p s>.20$. For P8 both main effects of ethnic group and task phase were not significant, $p s>.89$. Since the interaction 


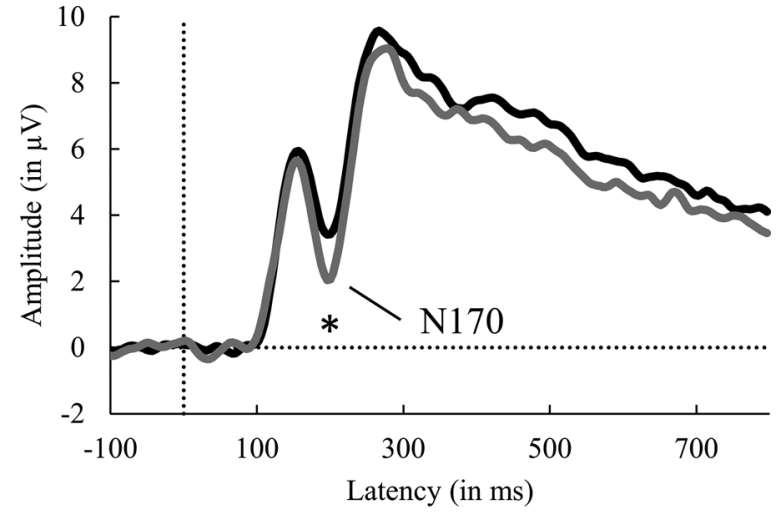

- Ingroup $\longrightarrow$ Outgroup

Fig. 3. Grand averaged ERP-waveforms at the left lateral parietal electrode for ingroup and outgroup child faces collapsed over both task phases. "significant difference in N170 amplitude at $\mathrm{p}<.05$.

Note: N170 components occurred as a negative deflation of the EEG signal, yet amplitudes were in the positive range. This was likely due to our choice of the average mastoid signal as the reference electrodes (see e.g. Joyce \& Rossion, 2005).

between ethnic group and electrode was qualified by task phase, additional pairwise comparisons were conducted to test if N170 amplitudes towards ingroup and outgroup child faces differed during the key pressing and cuteness ratings phases. This revealed no significant differences in N170 amplitudes during both tasks, all $p s>.13$.

\subsection{Correlations between ERPs and behavior}

Difference scores were calculated between amplitudes to ingroup and outgroup child faces for the N200 measured over Pz and the N170 measured over P7. For N200 difference scores, scores that are more positive indicate stronger N200s towards ingroup children compared to outgroup children. N170 difference scores with negative values indicated stronger responses towards outgroup child faces compared to ingroup children. Difference scores were correlated with behavioral results (see Table 1).

There was a significant negative correlation of N200 difference scores with cuteness ratings of outgroup child faces, $r=-.35, n=38$, $p=.03$, see Fig. 4a. This indicated that the more participants differentiated between ingroup and outgroup child faces in the N200, the less cute they rated outgroup child faces. N200 difference scores were not related to ratings of ingroup child faces, nor were there significant correlations between N200 difference scores and viewing times of the outgroup and ingroup children during the keypress phase, all $p s>14$. For the N170 difference scores there were no significant correlations with viewing times or cuteness ratings, all $p s>.33$.

PCAT scores were positively correlated with cuteness ratings of outgroup child faces, $r=.38, n=38, p=.02$, see Fig. $4 \mathrm{~b}$. This indicates that participants with increased motivation for parental care rated outgroup child faces as cuter. There was no correlation between PCAT scores and ratings of ingroup child faces, nor were there significant correlations between PCAT scores and viewing times of the ingroup and outgroup child faces, all $p s>.25$. Additionally, there was no significant correlation between PCAT scores and ERP contrast scores, both $p s>.27$. This indicates that individual differences in PCAT scores did not account for individual differences in ERP contrast scores.

\section{Discussion}

Ethnic disparities in educational and health care settings that put children of ethnic minority groups at a disadvantage have received considerable attention in research (Bates \& Glick, 2013; Flores, 2010; Glock et al., 2013; Rubie-Davies et al., 2006; Tenenbaum \& Ruck, 2007). The current study aimed to examine the underlying neural responses that may contribute towards implicit attitudes leading to these persistent inequalities. In line with the literature on intergroup differences in the processing of adult faces, Dutch Caucasian women displayed significant differences in electrophysiological activity towards child faces with characteristics of other ethnicities compared to children of their own ethnicity. Our results show that N200 components were increased in response to ingroup child faces compared to outgroup faces. This is in accordance with previous research using adult stimuli of different ethnicities, where enhanced N200 s components have been proposed to reflect a spontaneous greater attention allocation towards more familiar, or ingroup, individuals (Ito \& Bartholow, 2009). Furthermore, our findings indicated that N170 components were more enhanced towards outgroup child faces compared to ingroup child faces. These findings support the hypothesis that N170 s are larger to outgroup faces, reflecting increased recruitment of face-processing mechanisms to outgroup targets (Ito \& Bartholow, 2009). Moreover, increased differentiation in N200 towards ingroup and outgroup child faces was related to a decrease in cuteness appraisal of outgroup child faces, indicating that early attentional processes may predict implicit biased behavior.

The current results demonstrate that the intergroup bias which is present in the early automatic processing of adult faces extends to young child faces. Furthermore, correlations between behavioral measures and ERPs showed that increased differentiation in attentional processing (N200) of ingroup and outgroup children resulted in significantly reduced cuteness ratings of outgroup children. This is of great significance since attractive or cuter children are generally judged and treated more positively than unattractive or less cute children (Langlois et al., 2000). However, it should be noted that whereas the ethnicity bias in ERPs related to lower cuteness rating, overall children of other ethnicities were not judged to be less cute, and even elicited longer viewing times. These longer viewing times might be driven by a novelty effect of the ethnical outgroup faces in our Dutch sample. Furthermore, individual differences in motivation for parental care positively influenced the appraisal of outgroup child faces. Participants scoring higher in motivation for parental care judged outgroup children to be cuter, thereby displaying a reversal of the intergroup bias disadvantageous for outgroup children. Since motivation for parental care did not relate to differentiation in neural responses towards outgroup and ingroup children, it remains unclear through which processes parental motivation may specifically enhance cuteness appraisals of outgroup children. However, the effect of individual differences of participants on intergroup-biased responding indicates that it may be difficult to study effects of ethnicity on adult responsivity using solely behavioral

Table 1

Correlations between ERP difference scores, PCAT scores and behavioral results.

\begin{tabular}{|c|c|c|c|c|c|}
\hline & Viewing times IG child faces & Viewing times OG child faces & Cuteness ratings IG child faces & Cuteness ratings OG child faces & PCAT scores \\
\hline N200 Pz (OG - IG) & -.24 & -.16 & -.03 & $-.35^{*}$ & -.18 \\
\hline N170 P7 (OG - IG) & -.02 & .12 & -.03 & .16 & .10 \\
\hline PCAT scores & .05 & -.01 & .19 & $.38^{*}$ & \\
\hline
\end{tabular}

Note: ${ }^{*} p<.05 ; \mathrm{IG}=$ Ingroup, OG $=$ Outgroup. 

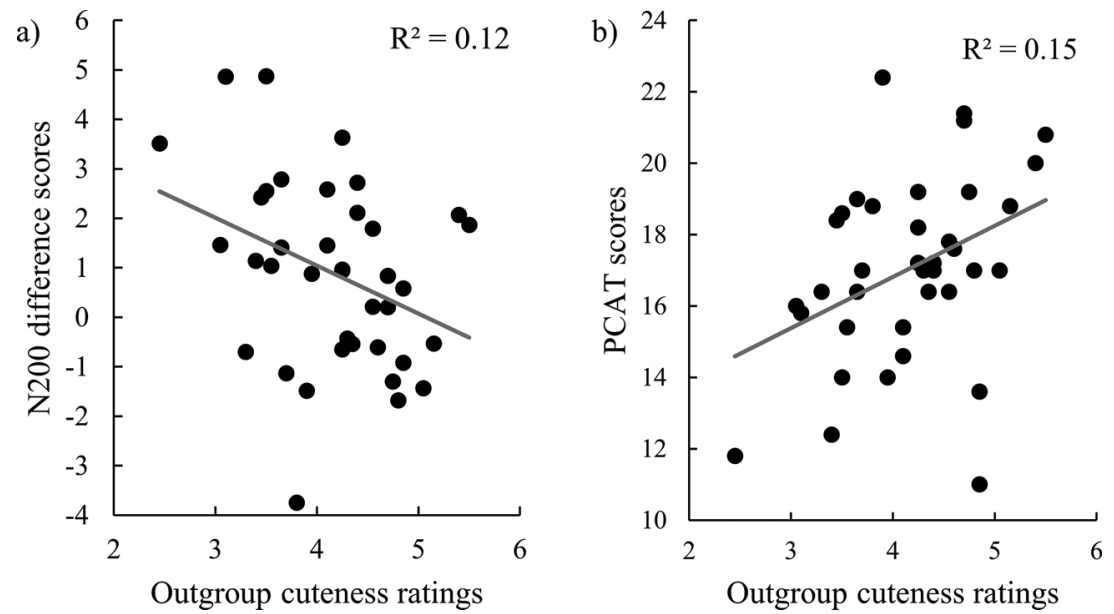

Fig. 4. Correlations between outgroup cuteness ratings and a) N200 difference scores, and b) PCAT scores.

measures. This may account for the fact that previous studies provided inconsistent findings in parental responsivity towards ingroup and outgroup infants (Esposito et al., 2014; Golle, Probst, Mast, \& Lobmaier, 2015; Hodsoll, Quinn, \& Hodsoll, 2010; Proverbio, De Gabrielle, Manfredi, \& Adorni, 2011) and highlights the importance of incorporating neurobiological measures (see also Bos, 2016). Similarly, Senese and colleagues (2017) showed that implicit associations to infant faces were influenced by genetic polymorphisms and early care experiences.

Unlike previous research, we did not find evidence for differential processing of ingroup and outgroup child faces measured with N100 and P200 components. One explanation for this discrepancy is that we adopted a lower number of trials per condition (40) compared to previous studies (e.g. 160 (Ito \& Urland, 2003) and 240 (Kubota \& Ito, 2007)). This could have made our experimental set-up less sensitive to detect intergroup differences in these very early attentional components. A similar finding and explanation was reported by Derks and colleagues (2015). An alternative explanation derives from the fact that our study is the first study to specifically study intergroup effects in ERP responses when facing children. Previous studies reported greater N100 and P200 components to outgroup members, suggesting a quick attentional direction towards more novel stimuli. Importantly, it has been proposed that this effect reflects a rapidly occurring vigilance towards (more threatening) outgroup members (Ito \& Bartholow, 2009). Since child stimuli are more likely to be perceived as appealing (as supposed to threatening) due to effects of baby schema (Luo et al., 2011) this may also explain why we did not find intergroup effects in the N100 and P200 components.

It should be noted that since the current study only focused on the processing of child faces, these findings do not confirm that the intergroup bias is the same for child and adult faces. Since previous studies have shown differential responses to adult and child faces, it is an avenue for future studies to further explore intergroup effects in children by directly comparing child and adult faces of different ethnicities. Furthermore, in order to examine how cuteness ratings directly reflect implicit attitudes that put children of ethnic minorities at a disadvantage in educational and health care settings, future studies should attempt to relate ERP responses to child faces of distinct ethnicities with explicit attitudes, such as judgements of personal abilities like intelligence or academic achievements, or implicit association tasks. This would lead to increased knowledge in the processes underlying social biased behavior towards children and would therefore generate greater understanding on how negative implicit biases could be reduced. For example, previous studies have shown that biases are learnt (Telzer, Humphreys, Shapiro, \& Tottenham, 2013) and that differences in neural responses to outgroup members may reduce and accuracy of outgroup facial recognition increases when intergroup contact increases (Chiroro \& Valentine, 1995; Walker et al., 2007). Furthermore, it has been shown that social biases can be modified by means of training (Lebrecht, Pierce, Tarr, \& Tanaka, 2009). Therefore, it would be of great interest for future research to collect data on individuals contact with outgroup members.

To the best of our knowledge, our study is the first to demonstrate that child faces with facial characteristics distinctive of different ethnicities are processed differently in adults. Electrophysiological findings are in line with what has previously been found for adult faces of different social groups. N200 components were enhanced in response to ingroup child faces compared to outgroup faces, while N170 s were stronger towards outgroup child faces compared to ingroup children reflecting differences in both early attentional and facial perceptual processing stages. Importantly, results revealed that increased differentiation in attentional processing (N200) for ingroup and outgroup children related to reduced cuteness ratings of outgroup children. Since judgements of cuteness can have great influence on how adults interact with children (Langlois et al., 2000), these findings contribute to the understanding of underlying mechanisms that result in ethnically biased treatment of children.

\section{Acknowledgements}

PAB was supported by a grant from the Netherlands Society of Scientific Research (451-14-015) and a Dynamics of Youth seed grant from Utrecht University.

\section{Appendix A. Supplementary data}

Supplementary material related to this article can be found, in the online version, at doi:https://doi.org/10.1016/j.biopsycho.2018.08. 015 .

\section{References}

Aharon, I., Etcoff, N., Ariely, D., Chabris, C. F., O'Connor, E., \& Breiter, H. C. (2001) Beautiful faces have variable reward value: fMRI and behavioral evidence. Neuron, 32(3), 537-551. https://doi.org/10.1016/S0896-6273(01)00491-3.

Balas, B., \& Nelson, C. A. (2010). The role of face shape and pigmentation in other-race face perception: An electrophysiological study. Neuropsychologia, 48(2), 498-506. https://doi.org/10.1016/j.neuropsychologia.2009.10.007.

Bates, L. A., \& Glick, J. E. (2013). Does it matter if teachers and schools match the student? Racial and ethnic disparities in problem behaviors. Social Science Research, 42(5), 1180-1190. https://doi.org/10.1016/j.ssresearch.2013.04.005.

Bornstein, M. H. (2015). Children's parents. In R. M. Lerner (Ed.). Handbook of child psychology and developmental science (pp. 1-78). (7th edition). Hoboken, NJ, USA: John Wiley \& Sons, Inc. https://doi.org/10.1002/9781118963418.

Bos, P. A. (2016). The endocrinology of human caregiving and its intergenerational 
transmission. Development and Psychopathology, 1-29. https://doi.org/10.1017/ S0954579416000973.

Buckels, E. E., Beall, A. T., Hofer, M. K., Lin, E. Y., Zhou, Z., \& Schaller, M. (2015). Individual differences in activation of the parental care motivational system: Assessment, prediction, and implications. Journal of Personality and Social Psychology, 108(1), 1-18. https://doi.org/10.1037/pspp0000023.

Caharel, S., Montalan, B., Fromager, E., Bernard, C., Lalonde, R., \& Mohamed, R. (2011). Other-race and inversion effects during the structural encoding stage of face processing in a race categorization task: An event-related brain potential study. International Journal of Psychophysiology, 79(2), 266-271. https://doi.org/10.1016/j. ijpsycho.2010.10.018.

Caldara, R., Rossion, B., Bovet, P., \& Hauert, C. (2004). Event-related potentials and time course of the "other-race" face classification advantage. Neuroreport, 15(3), 905-910. https://doi.org/10.1097/01.wnr.00001.

Caldara, R., Thut, G., Servoir, P., Michel, C., Bovet, P., \& Renault, B. (2003). Face versus non-face object perception and the "other-race" effect: A spatio-temporal event-related potential study. Clinical Neurophysiology, 114(3), 515-528. https://doi.org/10. 1016/S1388-2457(02)00407-8.

Chiroro, P., \& Valentine, T. (1995). An investigation of the contact hypothesis of the ownrace bias in face recognition. The Quarterly Journal of Experimental Psychology, 48(4), 879-894. https://doi.org/10.1080/14640749508401421.

Correll, J., Urland, G. R., \& Ito, T. A. (2006). Event-related potentials and the decision to shoot: The role of threat perception and cognitive control. Journal of Experimental Social Psychology, 42(1), 120-128. https://doi.org/10.1016/j.jesp.2005.02.006.

Derks, B., Stedehouder, J., \& Ito, T. A. (2015). Social identity modifies face perception: An ERP study of social categorization. Social Cognitive and Affective Neuroscience, 10(5), 672-679. https://doi.org/10.1093/scan/nsu107.

Eimer, M. (2011). The face-sensitive N170 component of the event-related brain potential. In A. J. Calder, G. Rhodes, M. H. Johnson, \& J. Haxby (Eds.). The Oxford Handbook of Face Perception (pp. 329-344). Oxford: Oxford University Press.

Esposito, G., Nakazawa, J., Ogawa, S., Stival, R., Kawashima, A., Putnick, D. L., .. Bornstein, M. H. (2014). Baby, you light-up my face: Culture-general physiological responses to infants and culture-specific cognitive judgements of adults. PloS One, 9(10), e106705. https://doi.org/10.1371/journal.pone.0106705.

Flores, G. (2010). Racial and ethnic disparities in the health and health care of children. Pediatrics, 125(4), e979-e1020. https://doi.org/10.1542/peds.2010-0188.

Glock, S., Krolak-Schwerdt, S., Klapproth, F., \& Böhmer, M. (2013). Beyond judgment bias: How students' ethnicity and academic profile consistency influence teachers' tracking judgments. Social Psychology of Education, 16(4), 555-573. https://doi.org/ 10.1007/s11218-013-9227-5.

Glocker, M. L., Langleben, D. D., Ruparel, K., Loughead, J. W., Valdez, J. N., Griffin, M. D., ... Gur, R. C. (2009). Baby schema modulates the brain reward system in nulliparous women. Proceedings of the National Academy of Sciences, 106(22), 9115-9119. https://doi.org/10.1073/pnas.0811620106.

Golle, J., Probst, F., Mast, F. W., \& Lobmaier, J. S. (2015). Preference for cute infants does not depend on their ethnicity or species: evidence from hypothetical adoption and donation paradigms. PloS One, 10(4), 1-19. https://doi.org/10.1371/journal.pone. 0121554.

Hahn, A. C., \& Perrett, D. I. (2014). Neural and behavioral responses to attractiveness in adult and infant faces. Neuroscience and Biobehavioral Reviews, 46(September), 591-603. https://doi.org/10.1016/j.neubiorev.2014.08.015.

Hahn, A. C., Symons, L. A., Kredel, T., Hanson, K., Hodgson, L., Schiavone, L., ... Jantzen, K. J. J. (2016). Early and late event-related potentials are modulated by infant and adult faces of high and low attractiveness. Social Neuroscience, 11(2), 207-220. https://doi.org/10.1080/17470919.2015.1059361.

Hahn, A. C., Xiao, D., Sprengelmeyer, R., \& Perrett, D. I. (2013). Gender differences in the incentive salience of adult and infant faces. The Quarterly Journal of Experimental Psychology, 66(1), 200-208. https://doi.org/10.1080/17470218.2012.705860.

Herrmann, M. J., Schreppel, T., Jäger, D., Koehler, S., Ehlis, A.-C., \& Fallgatter, A. J. (2007). The other-race effect for face perception: An event-related potential study. Journal of Neural Transmission, 114(7), 951-957. https://doi.org/10.1007/s00702007-0624-9.

Hewstone, M., Rubin, M., \& Willis, H. (2002). Intergroup Bias. Annual Review of Psychology, 53(1), 575-604. https://doi.org/10.1146/annurev.psych.53.100901. 135109.

Hildebrandt, K. A., \& Fitzgerald, H. E. (1983). The infant's physical attractiveness: Its effect on bonding and attachment. Infant Mental Health Journal, 4(1), 1-12. https:// doi.org/10.1002/1097-0355(198321)4:1 < 1::AID-IMHJ2280040102>3.0.CO;2-2.

Hodsoll, J., Quinn, K. A., \& Hodsoll, S. (2010). Attentional prioritization of infant faces is limited to own-race infants. PloS One, 5(9), 1-5. https://doi.org/10.1371/journal. pone.0012509.

Ito, T. A., \& Bartholow, B. D. (2009). The neural correlates of race. Trends in Cognitive Sciences, 13(12), 524-531. https://doi.org/10.1016/j.tics.2009.10.002.

Ito, T. A., \& Urland, G. R. (2003). Race and gender on the brain: Electrocortical measures of attention to the race and gender of multiply categorizable individuals. Journal of Personality and Social Psychology, 85(4), 616-626. https://doi.org/10.1037/0022 3514.85.4.616.

Ito, T. A., \& Urland, G. R. (2005). The influence of processing objectives on the perception of faces: An ERP study of race and gender perception. Cognitive, Affective \& Behavioral Neuroscience, 5(1), 21-36. https://doi.org/10.3758/CABN.5.1.21.

Ito, T. A., Thompson, E., \& Cacioppo, J. T. (2004). Tracking the timecourse of social perception: On event-related brain potentials. Personal and Social Psychological Bulletin, 30, 1267-1280. https://doi.org/10.1177/0146167204264335.
Jantzen, K. J., Symons, L. A., Kredel, T., Ratcliff, B., Toor, N., Jantzen, M. G., ... Hahn, A C. (2017). The spatiotemporal neural dynamics of infant face processing. Adaptive Human Behavior and Physiology, 3(4), 386-400. https://doi.org/10.1007/s40750017-0080-1.

Joyce, C., \& Rossion, B. (2005). The face-sensitive N170 and VPP components manifest the same brain processes: The effect of reference electrode site. Clinical Neurophysiology, 116(11), 2613-2631. https://doi.org/10.1016/j.clinph.2005.07. 005 .

Kringelbach, M. L., Lehtonen, A., Squire, S., Harvey, A. G., Craske, M. G., Holliday, I. E., ... Stein, A. (2008). A specific and rapid neural signature for parental instinct. PloS One, 3(2), e1664. https://doi.org/10.1371/journal.pone.0001664.

Kubota, J. T., \& Ito, T. A. (2007). Multiple cues in social perception: The time course of processing race and facial expression. Journal of Experimental Social Psychology, 43(5), 738-752. https://doi.org/10.1016/j.jesp.2006.10.023.

Langlois, J. H., Kalakanis, L., Rubenstein, A. J., Larson, A., Hauam, M., \& Smoot, M. (2000). Maxims or myths of beauty? A Meta-Analytic and Theoretical Review, 126(3), 390-423. https://doi.org/10.1037//0033-2909.126.3.390.

Langlois, J. H., Ritter, J. M., Casey, R. J., \& Sawin, D. B. (1995). Infant attractiveness predicts maternal behaviors and attitudes. Developmental Psychology, 31(3), 464-472.

Lebrecht, S., Pierce, L. J., Tarr, M. J., \& Tanaka, J. W. (2009). Perceptual other-race training reduces implicit racial bias. PloS One, 4(1), e4215. https://doi.org/10.1371/ journal.pone.0004215.

Lipp, O. V., Mallan, K. M., Martin, F. H., Terry, D. J., \& Smith, J. R. (2011). Electrocortical implicit race bias does not vary with participants' race or sex. Social Cognitive and Affective Neuroscience, 6(5), 591-601. https://doi.org/10.1093/scan/nsq089.

LoBue, V., \& Thrasher, C. (2015). The Child Affective Facial Expression (CAFE) set: Validity and reliability from untrained adults. Frontiers in Psychology, 5, 1-8. https:// doi.org/10.3389/fpsyg.2014.01532.

Lorenz, K. (2010). Die angeborenen Formen möglicher Erfahrung. Zeitschrift Für Tierpsychologie, 5(2), 235-409. https://doi.org/10.1111/j.1439-0310.1943. tb00655.x.

Luck, S. J. (2014). An introduction to the event-related potential technique. Cambridge, MA: The MIT Press.

Luo, L. Z., Li, H., \& Lee, K. (2011). Are children's faces really more appealing than those of adults? Testing the baby schema hypothesis beyond infancy. Journal of Experimental Child Psychology, 110(1), 115-124. https://doi.org/10.1016/j.jecp.2011.04.002.

Luo, L. Z., Ma, X., Zheng, X., Zhao, W., Xu, L., Becker, B., ... Kendrick, K. M. (2015). Neural systems and hormones mediating attraction to infant and child faces. Frontiers in Psychology, 6(970), 1-22. https://doi.org/10.3389/fpsyg.2015.00970.

Ofan, R. H., Rubin, N., \& Amodio, D. M. (2011). Seeing race: N170 responses to race and their relation to automatic racial attitudes and controlled processing. Journal of Cognitive Neuroscience, 23(10), 3153-3161. https://doi.org/10.1162/jocn_a_00014.

Parsons, C. E., Young, K. S., Kumari, N., Stein, A., \& Kringelbach, M. L. (2011). The motivational salience of infant faces is similar for men and women. PloS One, 6(5), 2-6. https://doi.org/10.1371/journal.pone.0020632.

Proverbio, A. M., De Gabrielle, V., Manfredi, M., \& Adorni, R. (2011). No race effect (ORE) in the automatic orienting toward baby faces: When ethnic group does not matter. Psychology, 2(9), 931-935. https://doi.org/10.4236/psych.2011.29140.

Ratner, K. G., \& Amodio, D. M. (2013). Seeing “us vs. them": Minimal group effects on the neural encoding of faces. Journal of Experimental Social Psychology, 49(2), 298-301. https://doi.org/10.1016/j.jesp.2012.10.017.

Rubie-Davies, C. M., Hattie, J., \& Hamilton, R. (2006). Expecting the best for students: Teacher expectations and academic outcomes. The British Journal of Educational Psychology, 76, 429-444. https://doi.org/10.1348/000709905X53589.

Senese, V. P., Shinohara, K., Esposito, G., Doi, H., Venuti, P., \& Bornstein, M. H. (2017) Implicit association to infant faces: Genetics, early care experiences, and cultural factors influence caregiving propensities. Behavioural Brain Research, 325, 163-172. https://doi.org/10.1016/j.bbr.2016.09.040.

Stahl, J., Wiese, H., \& Schweinberger, S. R. (2008). Expertise and own-race bias in face processing: An event-related potential study. Neuroreport, 19(5), 583-587. https:// doi.org/10.1097/WNR.0b013e3282f97b4d.

Telzer, E. H., Humphreys, K. L., Shapiro, M., \& Tottenham, N. (2013). Amygdala sensitivity to race is not present in childhood but emerges over adolescence. Journal of Cognitive Neuroscience, 25(2), 234-244. https://doi.org/10.1162/jocn_a_00311.

Tenenbaum, H. R., \& Ruck, M. D. (2007). Are teachers' expectations different for racial minority than for European American students? A meta-analysis. Journal of Educational Psychology, 99(2), 253-273. https://doi.org/10.1037/0022-0663.99.2. 253.

Vizioli, L., Foreman, K., Rousselet, G. A., \& Caldara, R. (2011). Inverting faces elicits sensitivity to race on the N170 component: A cross-cultural study. Journal of Vision, 10(1), 1-13. https://doi.org/10.1167/10.1.15.

Walker, P. M., Silvert, L., Hewstone, M., \& Nobre, A. C. (2007). Social contact and otherrace face processing in the human brain. Social Cognitive and Affective Neuroscience, 3(1), 16-25. https://doi.org/10.1093/scan/nsm035.

Wiese, H., Stahl, J., \& Schweinberger, S. R. (2009). Configural processing of other-race faces is delayed but not decreased. Biological Psychology, 81(2), 103-109. https://doi. org/10.1016/j.biopsycho.2009.03.002.

Willadsen-Jensen, E. C., \& Ito, T. A. (2015). The effect of context on responses to racially ambiguous faces: Changes in perception and evaluation. Social Cognitive and Affective Neuroscience, 10(7), 885-892. https://doi.org/10.1093/scan/nsu134.

Yamamoto, R., Ariely, D., Chi, W., Langleben, D. D., \& Elman, I. (2009). Gender differences in the motivational processing of babies are determined by their facial attractiveness. PloS One, 4(6), 4-7. https://doi.org/10.1371/journal.pone.0006042. 